\title{
Efeitos cardiovasculares do butorfanol na anestesia por romifidina/ midazolam/cetamina em gatos
}

\section{Cardiovascular effects of butorphanol in romifidine/midazolam/ketamine anesthesia in cats}

\author{
André Luis Selmi, * Guilherme Maia Mendes, ${ }^{\star \star}$ Glenda Ramalho Barbudo-Selmi, ${ }^{\star \star \star}$ \\ Juliana Peboni Figueiredo**
}

\begin{abstract}
Resumo
Estudaram-se os efeitos cardiovasculares induzidos pelo butorfanol em seis gatos anestesiados com romifidina/midazolam/ cetamina. Administrou-se por via intramuscular, ao grupo RMK, $0,04 \mathrm{mg} / \mathrm{kg}$ de romifidina, $0,1 \mathrm{mg} / \mathrm{kg}$ de midazolam e $8 \mathrm{mg} / \mathrm{kg}$ de cetamina e no grupo RMKB adicionou-se $0,2 \mathrm{mg} / \mathrm{kg}$ de butorfanol. Aferiram-se as freqüências cardíaca (FC) e respiratória (FR), pressão arterial sistólica (PAS), diastólica (PAD) e média (PAM), saturação parcial de oxi-hemoglobina $\left(\mathrm{SpO}_{2}\right)$ e temperatura retal (TR) durante 60 minutos. O grau de sedação, analgesia, miorrelaxamento, reflexo de endireitamento e resposta auditiva foram também subjetivamente avaliados. O grupo RMKB apresentou aumento no período hábil anestésico, melhor sedação e miorrelaxamento aos cinco minutos, analgesia aos dez minutos, resposta auditiva e recuperação do reflexo de endireitamento aos 60 minutos. A redução na FR foi similar, porém foi observada diminuição da $F C$, no grupo RMKB, com manutenção da pressão arterial. Conclui-se que a adição do butorfanol à associação romifidina/midazolam/cetamina prolonga o tempo e a qualidade de anestesia, porém reduz a freqüência cardíaca.
\end{abstract}

Palavras-chave: romifidina, butorfanol, midazolam, cetamina, anestesia, gatos.

\begin{abstract}
Cardiovascular effects of butorphanol were studied in six cats anesthetized with romifidine/midazolam/ketamine. Romifidine $(0,04 \mathrm{mg} / \mathrm{kg})$, midazolam $(0,1 \mathrm{mg} / \mathrm{kg})$ and ketamine $(8 \mathrm{mg} / \mathrm{kg})$ were administered at same time intramuscularly in RMK group, and butorphanol $(0,2 \mathrm{mg} / \mathrm{kg})$ was added to this protocol in RMKB group. Heart and respiratory rates, sistolic, diastolic and mean arterial pressure, pulse oximetry and rectal temperature were evaluated for 60 minutes. Sedation, analgesia, muscle relaxation, righting reflex and auditory response were subjectively evaluated. Mean anesthetic time was significantly longer in RMKB group. Degree of sedation and muscle relaxation at 5 minutes, analgesia at 10 minutes and auditory response at 60 minutes of anesthesia were significantly higher in RMKB group. A significant reduction in respiratory rate occurred in both groups. In RMKB group, heart rate was decreased while arterial pressure was maintained during anesthesia. Based on results obtained with this study, it was concluded that the addition of butorphanol on romifidine/midazolam/ketamine provides higher anesthetic level and prolonged anesthesia time, but bradycardia is a main side effect.
\end{abstract}

Keywords: romifidine, butorphanol, midazolam, ketamine, anesthesia, cats.

\section{Introdução}

A associação de anestésicos tem sido responsável pelo grande avanço da anestesia intravenosa de pequenos animais, promovendo analgesia trans e pós-operatória (Kehlet e Dahl, 1993; Pypendop et al., 1996). Assim, os agonistas a-2 adrenérgicos e os opióides estão entre os agentes anestésicos mais utilizados na analgesia operatória em cães e gatos (Pypendop et al., 1996; Cruz et al., 2000; Itamoto et al., 2000).
A romifidina, potente agonista a-2 adrenérgico, teve recentemente seus efeitos sedativos, analgésicos e miorrelaxantes avaliados em gatos (Cruz et al., 2000; Selmi et al., 2001). Em cães, ela promove efeitos cardiorrespiratórios similares aos demais agentes deste grupo (Klide et al., 1975; Haskins et al., 1986), ou seja, bradicardia, hipertensão transitória seguida de hipotensão, redução do volume sistólico e débito cardíaco, aumento da resistência vascular e depressão respiratória (Pypendop e Verstegen, 2001).

\footnotetext{
* Professor. Universidade Anhembi Morumbi. Curso de Medicina Veterinária. Rua Dr. Almeida Lima, 1134, Brás, CEP 03164-000 São Paulo, SP andré_selmi@yahoo.com.br

** Médico-veterinário. UnB/FAV.

** Doutorando. FCAV UNESP.
} 
O butorfanol é um opióide agonista-antagonista de uso freqüente na prática anestésica veterinária (Hosgood, 1990) e foi considerado três a cinco vezes mais potente que a morfina (Jaffe e Martin, 1990). Promove analgesia por ação agonista em receptores kappa e pouca interferência sobre os parâmetros respiratórios (Trim, 1983).

A associação de opióides e agonistas a-2 adrenérgicos aos benzodiazepínicos representa uma alternativa para a realização de procedimentos cirúrgicos de curta duração (Tranquilli et al., 1990; Verstegen e Petcho, 1993, Itamoto et al., 2000). O midazolam, um benzodiazepínico hidrossolúvel, produz sedação e relaxamento muscular, sendo usualmente empregado como medicação pré-anestésica (Gross et al., 1990; llkiw, 1992). O cloridrato de cetamina é um anestésico dissociativo que promove analgesia somática, além de hipertonia muscular, sialorréia e estimulação simpática, efeitos normalmente minimizados quando este agente é associado aos agonistas a-2 adrenérgicos (Haskins et al., 1986).

Considerando-se estes aspectos, objetivou-se estudar os efeitos cardiovasculares da anestesia com da romifidina, midazolam e cetamina, associados ao butorfanol em gatos.

\section{Materiais e métodos}

Foram utilizados seis gatos adultos, machos ou fêmeas, pesando entre 1,8 e $3,8 \mathrm{~kg}$, mantidos em jejum alimentar de 12 horas e hídrico de 4 horas. Eles foram dispostos em dois grupos: Grupo RMK, anestesiados com $0,04 \mathrm{mg} / \mathrm{kg}$ de romifidina $^{a} 0,1 \mathrm{mg} / \mathrm{kg}$ de midazolam ${ }^{b} 8 \mathrm{mg} / \mathrm{kg}$ de cetamina; ${ }^{c} \mathrm{e}$ Grupo RMKB, submetido ao mesmo tipo de anestesia, acrescida de $0,2 \mathrm{mg} / \mathrm{kg}$ de butorfanol. ${ }^{\mathrm{d}}$ Os fármacos foram associados em uma mesma seringa, sendo posteriormente administrados pela via intramuscular. Todos os animais foram anestesiados duas vezes, respeitando-se intervalo mínimo de três semanas entre cada associação anestésica.

Empregou-se um monitor multiparamétrico oscilométrico ${ }^{\circ}$ para mensuração dos valores basais de: pressão arterial sistólica (PAS); diastólica (PAD) e média (PAM), e ainda, a saturação parcial de oxigênio $\left(\mathrm{SpO}_{2}\right)$. A freqüência cardíaca $(\mathrm{FC})$ foi mensurada por meio de estetoscópio; a freqüência respiratória (FR) foi determinada pelo número de excursões torácicas durante um minuto e a temperatura retal foi registrada por meio de termômetro digital. Todos parâmetros foram avaliados no momento anterior às aplicações das associações anestésicas (momento 0) e aos 5, 10, 20, 30, 40, 50 e 60 minutos após administração dos fármacos.

Avaliou-se o tempo de latência (período de tempo entre a aplicação até a perda dos reflexos posturais), período de decúbito (considerado o intervalo entre a aplicação até o decúbito lateral dos animais), período de hipnose (tempo compreendido entre o decúbito até a primeira tentativa de levantar a cabeça alguns centímetros do solo), tempo de emergência anestésica (período de tempo entre o fim do tempo de anestesia e recuperação da postura quadrupedal) e período de recuperação

\footnotetext{
a Sedivet, Boehringer, Itapecerica da Serra, Brasil.

- Dormire, Criatália, Itapira, Brasil.

c Francotar, Francodex, São Paulo, Brasil.

- Torbugesic, Fort Dodge Laboratories Inc., lowa, USA.

- Dixtal - DX 2710, Manaus, Brasil.

' Statistical Analysis System, Cary, USA.
}

anestésica (intervalo entre o tempo de emergência até a completa deambulação dos animais).

Além destas variáveis, utilizou-se sistema quali-quantitativo de escore por números, conforme o Quadro 1, para avaliação dos seguintes parâmetros: sedação, miorrelaxamento, analgesia, resposta auditiva e reflexo de endireitamento.

Os dados referentes ao tempo de latência, período de decúbito, período de hipnose, tempo de emergência anestésica e recuperação anestésica foram analisados e comparados pelo teste $t$ de Student. As variáveis fisiológicas foram analisadas pelo ANOVA com medidas repetidas nos tempos em cada grupo e comparadas pelo teste de Tukey. Os dados referentes à sedação, miorrelaxamento, analgesia, resposta auditiva e reflexo de endireitamento foram avaliados por ANOVA para dados não-paramétricos seguido pelo teste de Kruskal-Wallis. As diferenças foram consideradas significativas quando $p<0,05$ e os resultados foram expressos em médias \pm desvios-padrão.

Quadro 1: Critérios adotados para a avaliação dos parâmetros anestésicos em gatos anestesiados com romifidina/ midazolam/cetamina (RMK) e romifidina/midazolam/cetamina/ butorfanol (RMKB)

\begin{tabular}{|l|}
\hline Sedação \\
O Acordado e andando \\
1 Capaz de andar, mas tropeça \\
2 Tenta, mas não fica de pé \\
3 Capaz de levantar a cabeça \\
4 Abre os olhos, mas não levanta a cabeça \\
5 Arresponsivo \\
Analgesia (pinçamento interdigital) \\
\hline Normal \\
1 leve \\
2 Moderado \\
3 Profundo \\
Miorrelaxamento (tônus mandibular e de membros) \\
\hline 0 Tônus muscular normal \\
1 Relaxamento muscular leve \\
2 Relaxamento muscular moderado \\
3 Relaxamento muscular profundo \\
Resposta auditiva (resposta a vigoroso bater de palmas \\
próximo aos ouvidos) \\
\hline 0 Resposta normal \\
1 Leve decréscimo de resposta (movimento de olhos e corpo) \\
2 Decréscimo moderado de resposta (movimento de olhos sem \\
movimento de corpo) \\
3 Ausência de resposta (sem movimento) \\
Reflexo de endireitamento (retorno à posição lateral após \\
posicionamento em decúbito dorsal) \\
\hline Resposta normal \\
1 leve decréscimo (reação produtiva com decréscimo motor) \\
\hline Ausposta (não há reação) \\
\hline
\end{tabular}




\section{Resultados}

Não foram observadas diferenças entre os grupos antes da administração das associações anestésicas para nenhum parâmetro.

Excetuando o período de hipnose hábil e o período de recuperação anestésica, estatisticamente maiores no grupo. RMKB, não ocorreram diferenças entre os demais tempos avaliados (Tabela 1).

Tabela 1: Médias e desvios-padrão dos tempos de latência, tempo de decúbito, período de hipnose hábil, tempo de emergência anestésica e período de recuperação anestésica em gatos anestesiados com romifidina/midazolam/cetamina (RMK) e romifidina/midazolam/cetamina/butorfanol (RMKB).

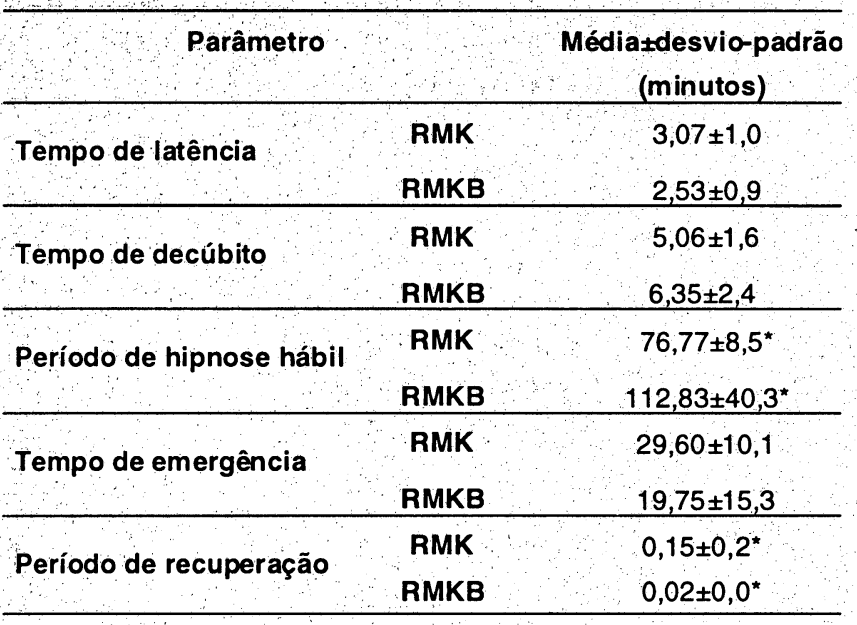

* Valor significativamente diferente entre os grupos $(p<0,05)$.

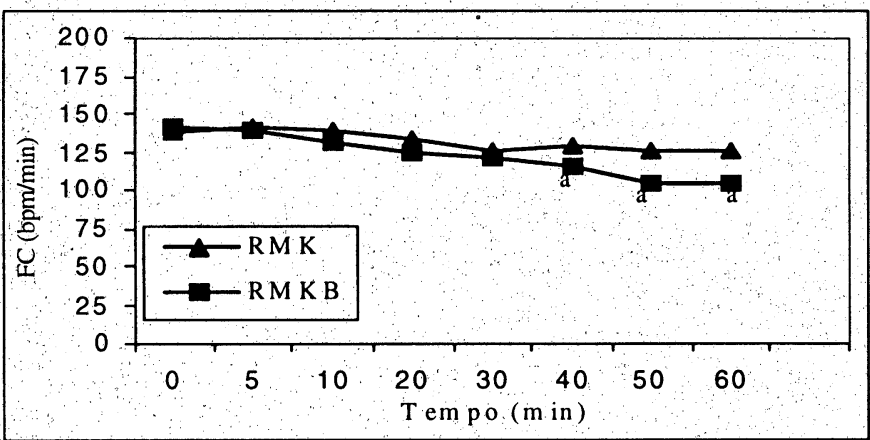

Valor significativamente diferente do valor basal $(p<0,05)$.

Figura 1: Médias da freqüência cardíaca ( $F C$ ) em gatos tratados com romifidina/midazolam/cetamina (RMK) ou romifidina/midazolam/ cetamina/butorfanol (RMKB).

Ao contrário da estabilidade da FC observada no grupo RMK, a adição do butorfanol à associação produziu decréscimo significativo deste parâmetro após 40 minutos de anestesia (Figura 1) Ambos protocolos promoveram decréscimo similar da FR após 20 minutos de anestesia (Figura 2).

Ocorreu diminuição da pressão arterial diastólica a partir dos 30 minutos e da pressão arterial média após 50 minutos no grupo RMK (Figura 3 ).
A temperatura retal e saturação parcial de oxi-hemoglobina se mantiveram estáveis em ambos os grupos durante o período de avaliação.

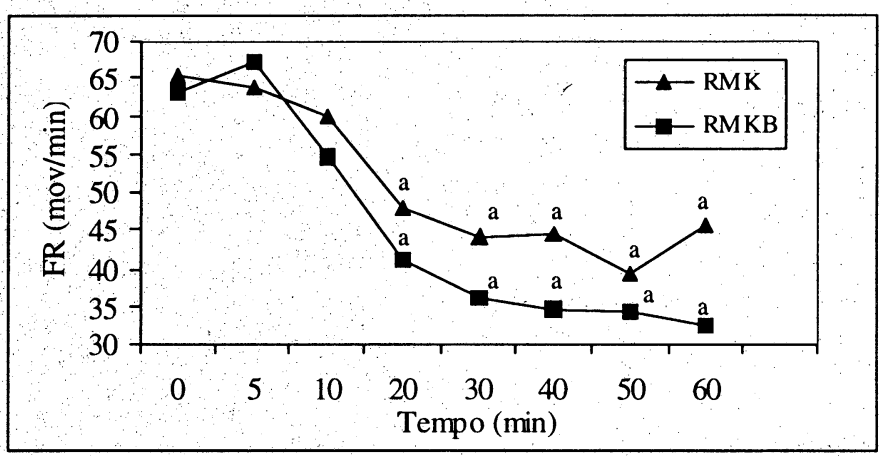

a Valor significativamente diferente do valor basal $(p<0,05)$.

Figura 2: Valores médios de freqüência respiratória em gatos tratados com romifidina/midazolam/cetamina (RMK) ou romifidina/midazolam/ cetamina/butorfanol (RMKB).

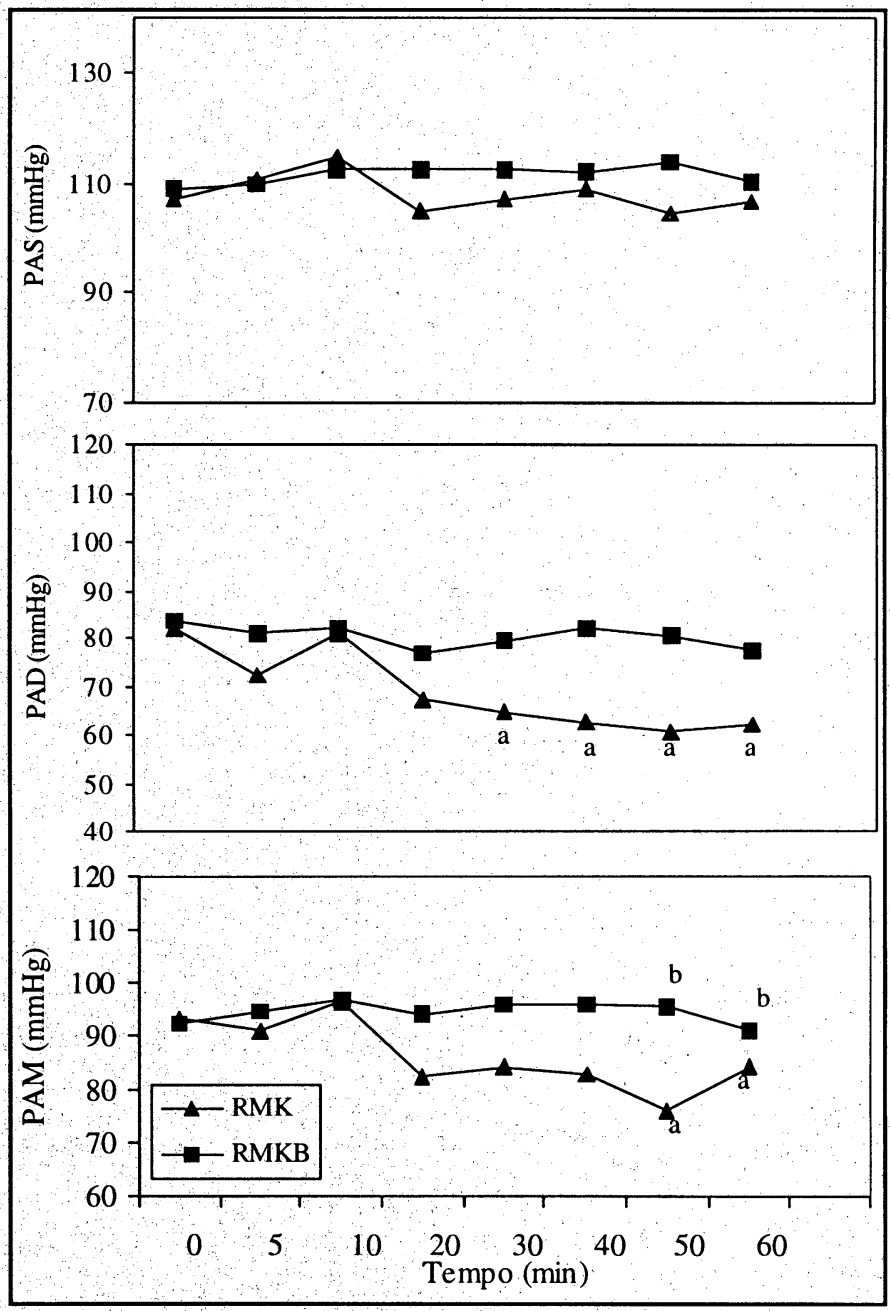

a Valor significativamente diferente do basal. ${ }^{b}$ Valor diferente do obtido no grupo RMK $(p<0,05)$.

Figura 3: Média dos valores de pressão arterial em gatos tratados com romifidina/midazolam/cetamina (RMK) ou romifidina/midazolam/ cetamina/butorfanol (RMKB). 
Além de prolongar o período de hipnose, a adição do butorfanol a associação RMK aumentou a sedação e miorrelaxamento aos cinco minutos e analgesia aos dez minutos de anestesia, não existindo diferenças durante os demais momentos. De maneira similar, no grupo RMKB foram obtidos melhores resultados do parâmetro reflexo de endireitamento aos cinco e 60 minutos. As avaliações qualitativas durante o período avaliado estão disponíveis na Tabela 2.

Tabela 2: Médias e desvios-padrão de sedação (SED), miorrelaxamento (MIO), analgesia (ANG), resposta auditiva (AUD) e reflexo de endireitamento (END) em gatos anestesiados com romifidina/midazolam/ cetamina (RMK) e romifidina/midazolam/cetamina/butorfanol (RMKB)

\begin{tabular}{|c|c|c|c|c|c|c|}
\hline Grupo & Tempo & SED & MIO & ANG & AUD & END \\
\hline \multirow[t]{8}{*}{ RMK } & Basal & $0,0 \pm 0,0$ & $0,0 \pm 0,0$ & $0,0 \pm 0,0$ & $0,0 \pm 0,0$ & $0,0 \pm 0,0$ \\
\hline & 5 & $2,1 \pm 1,6^{*}$ & $0,6 \pm 1,2$ & $0,4 \pm 0,4$ & $0,5 \pm 1,2$ & $0,5 \pm 1,2$ \\
\hline & 10 & $4,6 \pm 0,8$ & $2,5 \pm 1,2$ & $2,3 \pm 0,5$ & $2,5 \pm 1,2$ & $2,5 \pm 1,2$ \\
\hline & 20 & $4,6 \pm 0,8$ & $2,5 \pm 1,2$ & $2,5 \pm 0,5$ & $2,6 \pm 0,8$ & $3,0 \pm 0,0$ \\
\hline & 30 & $5,0 \pm 0,0$ & $3,0 \pm 0,0$ & $2,5 \pm 0,5$ & $3,0 \pm 0,0$ & $3,0 \pm 0,0$ \\
\hline & 40 & $5,0 \pm 0,0$ & $3,0 \pm 0,0$ & $2,1 \pm 0,3$ & $3,0 \pm 0,0$ & $3,0 \pm 0,0$ \\
\hline & 50 & $5,0 \pm 0,0$ & $3,0 \pm 0,0$ & $2,1 \pm 0,3$ & $2,6 \pm 0,5$ & $3,0 \pm 0,0$ \\
\hline & 60 & $5,0 \pm 0,0$ & $3,0 \pm 0,0$ & $1,5 \pm 0,2$ & $2,0 \pm 0,8$ & $2,1 \pm 0,9$ \\
\hline \multirow[t]{8}{*}{ RMKB } & Basal & $0,0 \pm 0,0$ & $0,0 \pm 0,0$ & $0,0 \pm 0,0$ & $0,0 \pm 0,0$ & $0,0 \pm 0,0$ \\
\hline & 5 & $3,8 \pm 0,4^{\circ}$ & $2,3 \pm 0,8^{\circ}$ & $0,3 \pm 0,3$ & $1,3 \pm 1,0$ & $2,3 \pm 1,2$ \\
\hline & 10 & $4,6 \pm 0,5$ & $2,8 \pm 0,4$ & $3,0 \pm 0,0^{\circ}$ & $2,5 \pm 1,2$ & $2,5 \pm 1,2$ \\
\hline & 20 & $5,0 \pm 0,0$ & $3,0 \pm 0,0$ & $2,8 \pm 0,1$ & $3,0 \pm 0,0$ & $3,0 \pm 0,0$ \\
\hline & 30 & $5,0 \pm 0,0$ & $3,0 \pm 0,0$ & $2,8 \pm 0,1$ & $3,0 \pm 0,0$ & $3,0 \pm 0,0$ \\
\hline & 40 & $5,0 \pm 0,0$ & $3,0 \pm 0,0$ & $2,1 \pm 0,3$ & $3,0 \pm 0,0$ & $3,0 \pm 0,0$ \\
\hline & 50 & $5,0 \pm 0,0$ & $3,0 \pm 0,0$ & $1,6 \pm 0,5$ & $3,0 \pm 0,0$ & $3,0 \pm 0,0$ \\
\hline & 60 & $5,0 \pm 0,0$ & $3,0 \pm 0,0$ & $1,6 \pm 0,5$ & $3,0 \pm 0,0^{\circ}$ & $3,0 \pm 0,0$ \\
\hline
\end{tabular}

* Valor significativamente diferente entre os grupos $(p<0,05)$.

\section{Discussão}

Associações anestésicas utilizando agonistas a-2 adrenérgicos, benzodiazepínicos e opióides tem demonstrado eficácia clínica na indução de anestesia cirúrgica em cães (Tranquilli et al., 1990; Verstegen e Petcho, 1993, Itamoto et al., 2000). A associação da cetamina a romifidina e midazolam, bem como a adição do butorfanol a este protocolo e os efeitos sobre os parâmetros cardiorrespiratórios ainda não tinham sido avaliados em gatos.

Um importante efeito sedativo do butorfanol foi evidenciado pelo aumento do período de hipnose hábil, corroborando com os achados de Tomizawa et al. (1997), England e Watts (1997) e Itamoto et al. (2000). A adição do butorfanol à associação da romifidina ao midazolam e cetamina aumentou 0 índice de anestesia, notados pela maior sedação, miorrelaxamento e analgesia, principalmente aos 10 minutos. A associação do butorfanol e romifidina na sedação de gatos mostrou-se eficaz (Selmi et al., 2001), e segundo England e Watts (1997), o butorfanol potencializa os efeitos sedativos e analgésicos da romifidina. Apesar do prolongamento do tempo de recuperação anestésica, observado no grupo RMKB, a utilização deste opióide em associações anestésicas poderá ser válida, por conferir melhor sedação e analgesia trans e pós-operatória, conforme relatado por Benson (1992) e Wagner (1999).

Os agonistas a-2 adrenérgicos induzem bradicardia (Klide et al., 1975; Cruz et al, 2000; Pypendop e Verstegen, 2001) e apesar do midazolam promover acréscimo da freqüência cardíaca (Jones et al., 1979; Gelman et al., 1983; Adams et al., 1985), esse efeito prevalece na associação destes. A estabilidade da freqüência cardíaca durante o período de anestesia, provavelmente, deveu-se à ação simpatomimética da cetamina (Haskins et al., 1986). Apesar do butorfanol acarretar mínimas alterações sobre 0 sistema cardiovascular (Trim, 1983; Hall e Clark, 1991), a adição deste agente diminuiu a freqüência cardiaca, possivelmente por efeito sinérgico sobre o tônus parassimpático quando associado ao agonista a-2 adrenérgico conforme obser vações realizadas por Selmi et al. (2000), sobrepondo-se aos efeitos simpáticos da cetamina. $A$ adição do butorfanol à associação de romifidina e tiletamina/zolazepam, em gatos, diminuiu a FC (Barbudo-Selmi et al., 2001), corroborando os achados deste estudo. Entretanto, naquele estudo, a FC diminuiu logo aos 20 minutos, sugerindo maiores riscos quando comparados aos resultados observados neste experimento.

Não foi observado o efeito bifásico, sobre a pressão arterial, característico da romifidina (Pypendop e Verstegen, 2001), que ocorre principalmente após administração intravenosa de agonistas a-2 adrenérgicos (Vainio e Palmu, 1989), talvez pelo fato da mensuração ter sido realizada posteriormente a este efeito. A manutenção da pressão arterial sistólica no grupo RMK ocorreu devido à estabilidade da freqüencia cardíaca durante a anestesia. O decréscimo significativo da pressão arterial diastólica após $30 \mathrm{mi}-$ nutos e da média a partir dos 50 minutos, neste grupo, possivelmente seja conseqüente da diminuição do tônus vascular periférico causado pela romifidina (Pypendop e Verstegen, 2001). Apesar de Itamoto et al, (2000) relatarem que a adição de butorfanol à associação medetomidina-midazolam resultou no aparecimento de hipertensão arterial em cães, estudos similares relatam que a hipotensão é o efeito mais pronunciado após a utilização destas associações (Tranquilli et al., 1990; Gross et al., 1993; Pypendop et al., 1996; Tomizawa et al., 1997). Todavia, a administração de romifidina-butorfanol em gatos manteve a pressão arterial estável (Selmi et al., 2001), o que contradiz os resultados obtidos em cães, explicando a estabilidade deste parâmetro no grupo RMKB.

Apesar da hipotermia ser um efeito freqüente durante a anestesia de pequenos animais, principalmente em decorrência da diminuição do trabalho muscular (Muir e Hubbell, 1992) e pela ação direta dos agonistas a-2 adrenérgicos so- 
bre o centro termorregulador no hipotálamo (Virtanen, 1989), não observou-se tal efeito neste estudo.

A depressão respiratória ocorreu de maneira similar em ambos os grupos. A diminuição da freqüência respiratória poderia ser oriunda da ação direta dos agonistas a-2 adrenérgicos no centro respiratório (Hall e Clarke, 1991), não existindo influência do butorfanol sobre este parâmetro, corroborando com citações que imputam aos agonistas kappa menor depressão respiratória do que os agonistas $m u$ (Raffe e Tranquilli, 1989; Hall e Clarke, 1991). Recentemente, Barbudo-Selmi et al. (2001) descreveram que a associação de tiletamina e zolazepam com a romifidina e butorfanol, em ga-

\section{Referências}

ADAMS, P.; GELMAN, S.; REVES, J.G., etal. Midazolam phamacodynamics and pharmacokinetics during acute hypovolemia. Anesthesiology. v. 63, p. 140-146, 1985.

BARBUDO-SELMI, G.R.; SELMI, A.L.; MELO, R.Q.; MARTINS, C.S.; MCMANUS, C. Efeitos cardiovasculares da associação romifidina/ butorfanol/tiletamina/zolazepam em gatos. Ciência Animal. v. 11, suplemento 1, p. 135, 2001.

BENSON, G.J. Assessment of analgesia by catecholamine analysis: response to onychectomy in cats. In: SHORT, C.E.; VAN POZNAK, A. (Ed.). Animal Pain. New York: Churchill Livingstone, 1992, p. 436-439.

CRUZ, M.L.; LUNA, S.P.; DE CASTRO, G.B.; et al. A preliminary trial comparison of several anesthetic techniques in cats. Canadian Veterinary Journal. v. 41, n. 6, p. 481-485, 2000.

ENGLAND, G.C.; WATTS, N. Effect of romifidine and romifidinebutorphanol for sedation in dogs. Journal of Small Animal Practice. v. 38, n.12, p. 561-564, 1997.

GELMAN, S.; REVES, J.G.; HARRIS, D. Circulatory responses to midazolam anesthesia: emphasis on canine splanchnic circulation. Anesthesia and Analgesia. v. 62, p. 135-139, 1983.

GROSS, M.E.; TRANQUILLI, W.J.; THURMON, J.C.; et al. Hemodinamic effects of intravenous midazolam-xylazine-butorphanol in dogs. Veterinary Surgery. v. 19, n. 2, p. 173-180, 1990.

HALL, L.W.; CLARKE, K.W. Principles of sedation, analgesia and premedication. In: HALL, L.W.; CLARKE, K.W. (Ed.). Veterinary Anesthesia. 9. ed. London: Bailliere Tindall, 1991, p. 51-79.

HASKINS, S.C.; PATZ, J.D.; FARVER, T.B. Xylazine and xylazineketamine in dogs. American Journal of Veterinary Research. v. 47, $n$. 3, p. 636-641, 1986.

HOSGOOD, G. Pharmacology features of butorphanol in dogs and cats. Journal of the American Veterinary Medical Association. v. 196 n. 1, p. 135-136, 1990.

ILKIW, J.E. Other potentially usefull new injectable anesthetic agents. Opnions in small animal anesthesia. Veterinary Clinics of North America - Small Animal Practice. v. 22, n. 2, p. 281-289, 1992.

ITAMOTO, K., HIRASA, Y., SAKONJYU, I., et al. Anaesthetic and cardiopulmonary effects of balanced anaesthesia with medetomidinemidazolam and butorphanol in dogs. Journal of Veterinary Medicine. $v$. 47 , n. 7, p. 411-420, 2000 .

JAFFE, J.H.; MARTIN, W.R. Opioid analgesics and antagonists. In: Goodman Gilman, A.; RALL, T.W.; NIES, A.S.; et al. Goodman and Gilman's the pharmacological basis of therapeutics. $8^{\text {th }}$ ed. New York: Pergamon Press, 1990, p. 485-521.

JONES, D.J.; STEHLING, L.C.; ZAUDER, H.L.Cardiovascular responses to diazepam and midazolam maleate in dog. Anesthesiology. v. 51, p. 430-434, 1979. tos, diminuiu pronunciadamente a FR, possivelmente pela ação do butorfanol, confirmando os achados deste estudo. Apesar da estabilidade observada nos valores de oximetria em ambos os grupos, entende-se que estudos sobre as pressões parciais dos gases sangüineos poderiam melhor elucidar as alterações respiratórias.

Conclui-se, com base nos resultados apresentados, que as associações propostas deprimem a FC e FR, e que embora o butorfanol aumente o período de hipnose hábil, a sedação, a analgesia e miorrelaxamento, causa depressão da freqüência cardíaca.

KEHLET, H.; DAHL, J.B. The value of multimodal or balanced analgesia in postoperative pain treatment. Anesthesia and Analgesia. v. 77, p. 1.048-1.056, 1993.

KLIDE, A.M.; CALDERWOOD, H.W.; SOMA, L.R. Cardiopulmonary effects of xylazine in dogs. American Journal of Veterinary Research. v. 36, n. 4, p. 931-935, 1975.

MUIR, W.W.; HUBBEL, J.A.E. Patient monitoring during anesthesia. In: MUIR, W.W.; HUBBEL, J.A.E. (Ed.). Handbook of Veterinary Anesthesia. 2. ed. St. Louis: Mosby, 1995, p. 165-172.

PYPENDOP, B.; SERTEYN, D.; VERSTEGEN, J. Hemodynamic effects of medetomidine-midazolam-butorphanol and medetomidine-midazolambuprenorphine combinations and reversibility by atipamezole in dogs. American Journal of Veterinary Research. v. 57, n. 5, p. 724-730, 1996. PYPENDOP, B.H.; VERSTEGEN, J.P. Cardiovascular effects of romifidine in dogs. American Journal of Veterinary Research. v. 62, n. 4, p. 490495, 2001.

RAFFE, M.R.; TRANQUILLI, W.J. Classifying commonly used analgesic agents. Veterinary Medicine. v. 89, p. 687-690, 1989.

SELMI, A.L.; MOREIRA, C.F.; MARTINS, C.S.; BARBUDO-SELMI, G.R.; MCMANUS, C. Efeitos cardiovasculares e sedativos da associação romifidina/butorfanol em gatos pré-tratados ou não pela atropina. $\mathrm{Ci}$ ência Animal. v. 11, suplemento 1, p. 144, 2001.

TOMIZAWA, N., TOMITA, I., NAKAMURA, K., et al. A comparative study of medetomidine-butorphanol and medetomidine-ketamine anaesthesia in dogs. Zentralblatt füe Veterinarmedizin Reihe. v. 44, n. 4, p. 189194, 1997.

TRANQUILLI, W.J.; GROSS, M.E.; THURMON, J.C.; et al. Evaluation of three midazolam-xylazine mixtures. Preliminary trials in dogs. Veterinary Surgery. v. 19, n. 2, p. 168-172, 1990.

TRIM, C.M.: Cardiopulmonary effects of butorphanol tartrate in dogs. Am J Vet Res. v. 44, n. 2, p. 329-331, 1983.

VAINIO, O.; PALMU, L. Cardiovascular and respiratory effects of medetomidine in dogs and influence of anticholinergics. Acta Veterinaria Scandinavica. v. 30, n. 4, p. 401-408, 1989.

VERSTEGEN, J.;PETCHO, A. Medetomidine-butorphanol-midazolam for anaesthesia in dogs and its reversal by atipamezole. Veterinary Record. v. 132, n. 5, p. $353-357,1993$.

VIRTANEN, R. Pharmacological profiles of medetomidine and its antagonist, atipamezole. Acta Veterinaria Scandinavica. Suplem. 85, p. 29-37, 1989.

WAGNER, A.E. Is butorphanol analgesic in dogs and cats? Veterinary Medicine. v. 94, n. 4, p. 346-351, 1999. 